\title{
Presentation and outcome of carpal tunnel syndrome with mini incision open carpal tunnel release
}

\author{
Muhammad Saaiq* (1) \\ Received: 20 Sep 2020 \\ Published: 26 May 2021
}

\begin{abstract}
Background: To document the clinical presentation of carpal tunnel syndrome (CTS) and evaluate the outcome of mini-incision open carpal tunnel release in terms of surgical complications, relief/persistence of symptoms at 4 months, and recurrence at 1 year.

Methods: This prospective case series included patients of all genders and ages who presented with CTS. They underwent release of transverse carpal ligament (TCL) under local anesthesia and tourniquet control on day care basis. Mini-incision of $2 \mathrm{~cm}$ to $2.5 \mathrm{~cm}$ was employed. The data were subjected to statistical analysis using SPSS version 21 (SPSS Inc). The percentages of key categorical variables were compared by employing the chi square test, and a $P$ value of less than .05 was regarded as statistically significant.

Results: There were 67 patients with 77 CTS affected wrists. A Significant majority of the patients $(\mathrm{n}=54 ; 80.59 \%)$ were females, whereas $13(19.40 \%)$ patients were males. The age range was 26 to 69 years, with a mean age of $41.01 \pm 11.70$ years. The CTS was found to be moderate among $34(44.15 \%)$ and severe among $43(55.84 \%)$ patients. The majority of patients had no operative complications. At 4 months follow-up, the majority of patients $(\mathrm{n}=74 ; 96.10 \%)$ reported symptomatic relief, whereas $3(3.89 \%)$ patients continued to have persistent severe symptoms. All the cases with persistent symptoms had diabetes mellitus of more than 5 years duration. There was no case of recurrence at one year follow-up.

Conclusion: CTS predominantly affected women aged 30 to 45 years. The open carpal tunnel release with mini-incision yielded good results in terms of relieving symptoms among the majority of patients and was associated with no critical complications or recurrence at 1 year.
\end{abstract}

Keywords: Carpal tunnel syndrome, Transverse carpal ligament release, Open carpal tunnel release, Compressive neuropathies

Conflicts of Interest: None declared

Funding: None

*This work has been published under CC BY-NC-SA 1.0 license.

Copyright $\odot$ Iran University of Medical Sciences

Cite this article as: Saaiq M. Presentation and outcome of carpal tunnel syndrome with mini incision open carpal tunnel release. Med J Islam Repub Iran. 2021 (26 May);35:67. https://doi.org/10.47176/mjiri.35.67

\section{Introduction}

Carpal tunnel syndrome (CTS) represents the most commonly encountered compressive neuropathy. It results from median nerve compression within the carpal tunnel. The classic symptoms inclu de paresthesia particularly at night, affecting the radial 3.5 digits of the hand; tingling

Corresponding author: Dr Muhammad Saaiq, muhammadsaaiq5@gmail.com

Department of Plastic surgery, National Institute of Rehabilitation Medicine (NIRM), Islamabad, Pakistan sensation, numbness or pain along these digits and progressive weakness of hand the grip. It is frequently bilateral; however, the dominant hand is usually more severely symptomatic $(1,2)$.

The condition accounts for $90 \%$ of all nerve entrapment

$\uparrow$ What is “already known” in this topic:

A variety of surgical approaches have been employed to address carpal tunnel syndrome (CTS). Each technique has its attended advantages and disadvantages. The mini-incision technique has recently emerged as promising one among them.

\section{$\rightarrow$ What this article adds:}

Careful initial evaluation and meticulous surgical decompression of the carpal tunnel with mini-incision provides predictably satisfactory outcome. Patients with diabetes mellitus have more chance of persistent symptoms despite adequate decompression. Hence, they should be counselled preoperatively so as to tailor their expectations accordingly. 
syndromes in the body, affecting approximately $1 \%$ to $4 \%$ of the general population. The treatment options range from conservative measures to open surgical release of the carpal tunnel. The initially instituted conservative treatment measures include wrist splinting, activity modification, vitamin B6 and B12 supplementation, non-steroidal anti-inflammatory agents, and local corticosteroid injections. The definitive treatment options range from endoscopic to open surgical release of the transverse carpal ligament (TCL) (1-3).

The current study was undertaken to document the clinical and demographic presentation of CTS among our patients and the evaluate the outcome of mini-incision open carpal tunnel release in terms of surgical complications, relief/persistence of symptoms at 4 months and recurrence at 1 year.

\section{Methods}

This case series study was performed at the National Institute of Rehabilitation Medicine (NIRM) Islamabad over a period of 3.5 years. (October 2016 to March 2020). Informed consent was taken from the patients. The study followed the ethical protocols as per Helsinki's Declaration-2013 revision. The study proceeded after approval by the hospital ethics committee via IRB/R/377.

\section{Inclusion Criteria}

The study included patients of all genders and ages who presented with CTS.

\section{Exclusion Criteria}

The exclusion criteria included patients with polyneuropathy, brachial plexopathy, cervical radiculopathy, median neuropathy proximal to the wrist level, musculoskeletal disorders, skeletal trauma and cases of gestational CTS.

\section{Diagnostic Criteria}

The diagnosis of CTS was based on the established clinical criteria and confirmed on electrodiagnostic studies (EDS) $(4,5)$. The clinical diagnostic criteria included the following: (1) paresthesia in the median nerve territory of the hand; (2) nocturnal paresthesia; (3) thenar region atrophy; (4) positive Tinel's test; (5) positive Phalen's test; and (6) decreased sensitivity (e.g. 2-point discrimination) in the median nerve innervated areas of the hand. In the EDS, slowed conduction velocities and prolongation of distal latencies along the median nerve were confirmed. The CTS was considered mild if EDS showed prolonged sensory latencies with normal motor studies. It was moderate CTS if the sensory abnormalities were coupled with prolonged motor distal latencies in the median nerve. The condition was labelled severe CTS when the aforesaid findings were coupled with an evidence of axonal loss also.

\section{Surgical Procedure}

All the surgical operations entailed complete release of the TCL. They were performed under infiltration anesthesia using lignocaine. Forearm tourniquet was employed.

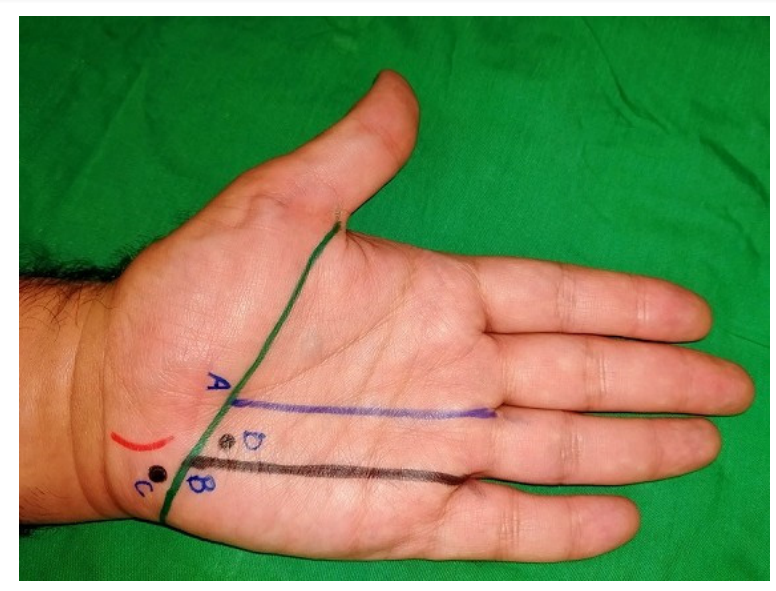

Fig. 1. Illustration of the Kaplan's lines. (a) The green color line shows the classic Kaplan's cardinal line, which is drawn vertically from the ulnar aspect of the extended thumb (first web space) towards the ulnar volar border of the wrist passing just distal to Pisiform bone. The superficial palmar arch courses distal to this line. (b) The blue color line and the point A: It is a perpendicular line drawn from the ulnar border of the middle finger and intersects the green Kaplan's cardinal line at point A. The point A indicates the point of emergence of the recurrent motor branch of median nerve. (c) The black color line and point B: It is the second perpendicular line drawn from the ulnar border of the ring finger that transects the Kaplan's cardinal line at point B. The point B represents the point of emergence of the motor branch of ulnar nerve. (d) The point $\mathrm{C}$ represents the underlying Pisiform bone. (e) The point D shows the underlying hook of Hamate. (f) The red color curvilinear line shows the mini-incision employed for the open carpal tunnel release.

The patient was positioned supine on the operating table with the arm positioned comfortably in $90^{\circ}$ abducted position on a Mayo's stand. The forearm was kept supinated. The wrist was kept $60^{\circ}$ extended with the help of a folded sterile gown placed under the dorsal aspect of the wrist. The incision site was marked, using the Kaplan's cardinal lines as a guide (6). A curvilinear mini-incision of $2 \mathrm{~cm}$ to $2.5 \mathrm{~cm}$ length was employed, located $6 \mathrm{~cm}$ to $10 \mathrm{~mm}$ ulnar to the thenar crease (Fig. 1). The skin, subcutaneous tissue and superficial palmar fascia were divided along the line of incision. The incision was deepened down to the TCL, which was divided on the most ulnar aspect of the median nerve. Initially a rent was carefully made in the TCL with size 15 surgical blade. A small fine tipped artery forceps was introduced to protect the underlying median nerve. The TCL was sharply divided with surgical blade, keeping the sharp edge outwards from the contents of the carpal tunnel. The TCL was divided in a proximalodistal fashion, through its entire length, both proximally as well as distally. Proximally the distal portion of the ante brachial fascia was also divided. The skin incision was closed with Prolene 4/0 interrupted sutures. (Fig. 2 A, B, and C) Sterile gauze dressing was applied. Sutures were removed 14 days after operation. Range of motion exercises of the fingers were encouraged on the third postoperative day. Night time wrist splint was advised for 3 weeks. The patient was encouraged to resume routine activities of daily living subsequently. The patients who had bilateral CTS underwent the operation for the contralateral side after 3 months interval. 

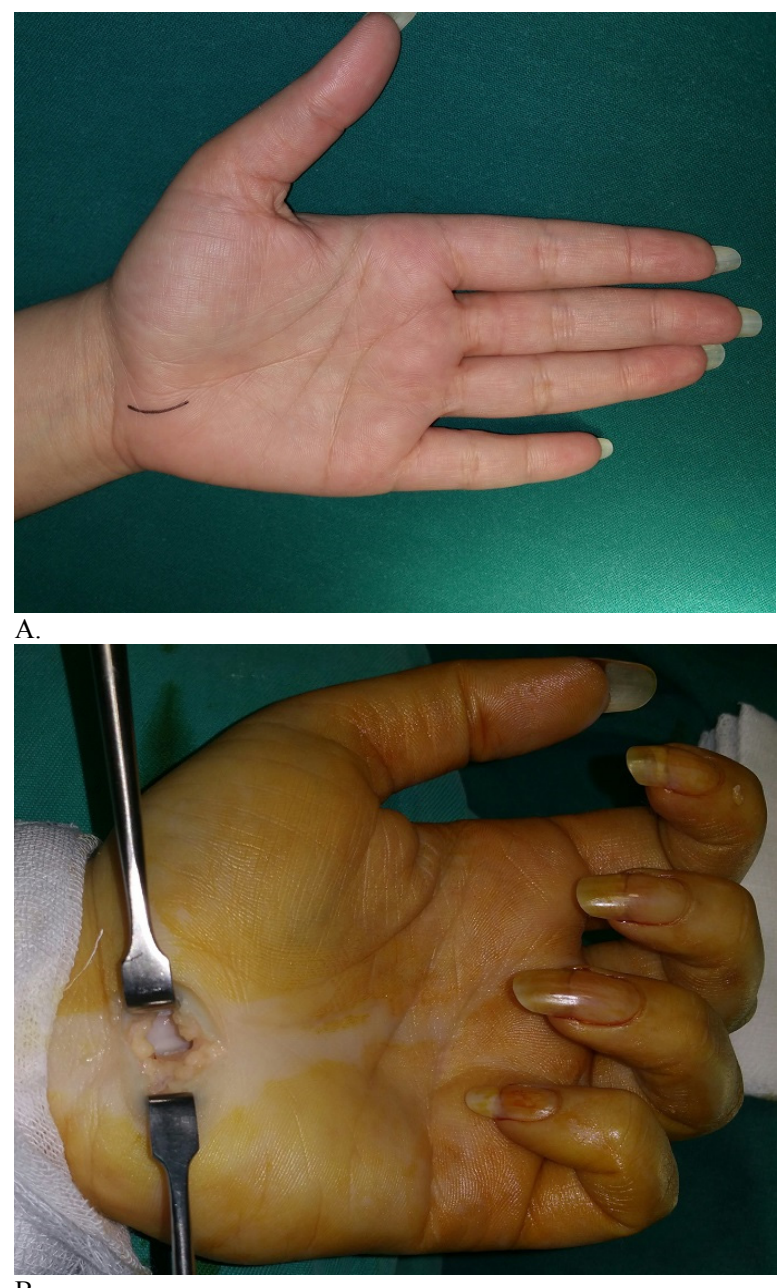

B.

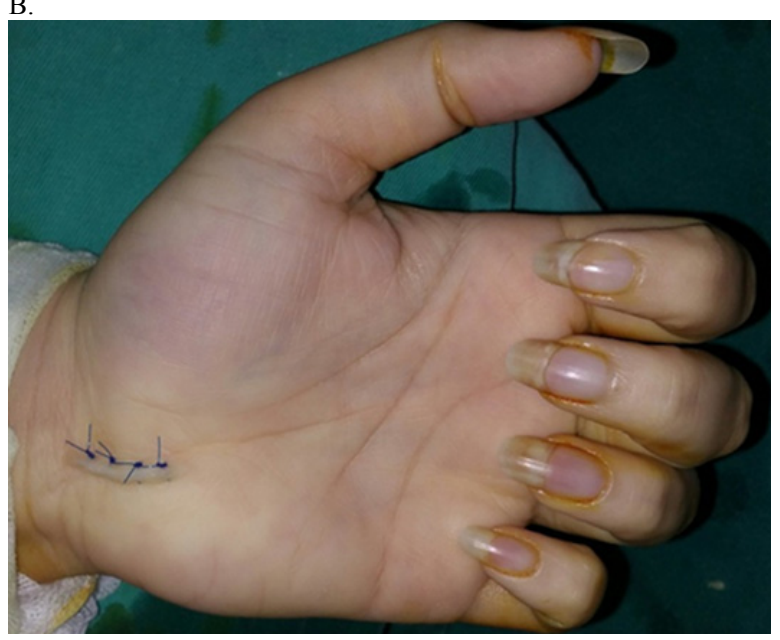

C.

Fig. 2. A patient with right sided CTS. (A) The curvilinear miniincision employed for open carpal tunnel release is indicated as the red line. (B) Intraoperative photograph showing the released TCL and the decompressed median nerve and carpal canal. (C) The incision is closure with prolene $4 / 0$ interrupted sutures.

\section{Statistical Analysis}

The demographic profile of the patients and outcomes were all recorded. The data were subjected to statistical analysis using SPSS version 21 (SPSS Inc). The outcome measures analyzed included any intraoperative and postoperative complications, relief or persistence of symptoms at 4 months, and recurrence at 1 year follow-up. The complications included intra-operative neurovascular and tendon injuries, postoperative wound-related complications, such as hematoma formation, infection, dehiscence, delayed healing, superficial epidermolysis at the incision margins, scar tenderness, scar hypertrophy and pillar pain. The preoperative and 4 months postoperative scores of modified Boston carpal tunnel questionnaire (BCTQ) were employed to assess relief versus persistence of symptoms $(7,8)$. Each patient answered a selfadministered questionnaire, entailing an overall 14 questions, using a Likert scale, ranging from 1 (mildest/no symptoms) to 5 (most severe symptoms). Thus, the minimum score for an individual patient was 14 , whereas the maximum was 70. In total, the questionnaire included the Symptom Severity Scale (SSS) and the Functional Status Scale (FSS). The SSS included 6 questions regarding severity of pain, paresthesia, numbness, weakness, nocturnal symptoms, and the over-all functional capacity. The FSS included questions regarding the following 8 functional activities: writing, buttoning of clothes, holding a book while reading, holding a phone while calling, opening a jar, performing household chores, carrying grocery bags, and bathing/dressing. For convenience of analysis, the resultant scores were arbitrarily stratified into mild (score $<30)$, moderate (30-49) and severe (50-70) categories.

Recurrence was defined as the re-appearance of the typical symptoms of CTS after surgery as measured at 1 year follow-up.

The percentages of key categorical variables were compared by employing the chi square test and a $\mathrm{P}$ value of less than .05 was regarded as statistically significant.

\section{Results}

There were 67 patients with 77 CTS affected wrists who underwent open carpal tunnel release. Ten patients underwent surgery for bilateral CTS.

A significant majority of the patients $(n=54 ; 80.59 \%)$ were females, whereas $13(19.40 \%)$ patients were males. The age ranged from 26 years to 69 years with a mean age of $41.01 \pm 11.70$ years.

The CTS was dominant-sided among 49 (63.6\%) patients. There was non dominant-sided involvement among $18(23.37 \%)$ patients whereas bilateral involvement was among $10(12.98 \%)$ patients $(\mathrm{p}<0.001)$.

The duration between the onset of symptoms and presentation for surgery ranged between 3 months to 17 months. The mean was $8.94 \pm 5.58$ months.

The majority of the patients had no known risk factors. Among the underlying associated factors, diabetes mellitus was found among $5(6.49 \%)$ patients and hypothyroidism in $1(1.29 \%)$ patient. The associated pathologic conditions found included trigger fingers in $6(7.79 \%)$ patients, de Quervain's disease in $2(2.59 \%)$ patients, and bilateral medial epicondylitis in $1(1.29 \%)$ patient.

On EDS, the CTS was found to be moderate in 34 $(44.15 \%)$ patients and severe in $43(55.84 \%)$. 
A significant majority of the patients had no complications $(p<0.00)$. There were no intraoperative complications. The postoperative complications encountered included superficial epidermolysis at the incision margins/ delayed wound healing $(n=7 ; 9.09 \%)$, scar tenderness $(\mathrm{n}=6 ; 7.79 \%)$, pillar pain $(\mathrm{n}=5 ; 6.49 \%)$ and scar hypertrophy $(n=2 ; 2.59 \%)$. All these improved with conservative measures within four months.

At 4 months follow-up, the majority of patients $(n=74$; $96.10 \%$ ) reported improved symptomatic relief, whereas 3 $(3.89 \%)$ patients continued to have persistent severe symptoms. All these patients with persistent symptoms had diabetes mellitus of more than 5 years duration. There was no case of recurrence at one year follow up.

Table 1 summarizes the various presenting characteristics of the patients and the outcomes observed among them.

\section{Discussion}

In this study, the majority of cases were aged between 30 to 40 years. Published studies have variably reported the affected age groups in their specific populations. For instance, $\mathrm{Xu} \mathrm{L}$ et al from China reported a mean age of 41 years with a range of 27 to 55 years, whereas Keser $\mathrm{N}$ et al from Turkey reported the mean age to be 55 years, with a range of 35 to 83 years $(9,10)$.

In this study predominant involvement of female patients was observed. Several published papers are akin to this observation. The higher frequency among women has been attributed to a variety of factors such as the anatomically narrower carpal tunnel being prone to higher internal pressures causing median nerve compression (9-12).

In this study the mean duration between onset of symptoms and presentation for surgical treatment was 8.94 months. Serna IC et al reported the mean duration between onset of symptoms and surgery as 5.86 months, with a range of 3 to 8 months (11).

In this study the majority of patients belonged to the idiopathic group with no known underlying risk factors for developing CTS. The risk factors for developing CTS may fall into one of the following principal categories: (1) extrinsic factors that increase the volume within the carpal tunnel; for example, myxedema, and pregnancy-associated increased body volume; (2) intrinsic factors within the

Table 1. Summary of the Presentation and Outcome Data $(\mathrm{n}=77)$

\begin{tabular}{|c|c|}
\hline Characteristics & Number of Patients (\%) \\
\hline $\begin{array}{l}\text { Gender: } \\
\text { Female } \\
\text { Male }\end{array}$ & $\begin{array}{l}54(80.59 \%) \\
13(19.40 \%)\end{array}$ \\
\hline $\begin{array}{l}\text { Side affected: } \\
\text { Dominant } \\
\text { Non-dominant } \\
\text { Bilateral }\end{array}$ & $\begin{array}{c}49(63.6 \%) \\
18(23.37 \%) \\
10(\%)\end{array}$ \\
\hline $\begin{array}{l}\text { Medical comorbidities and Associated conditions: } \\
\text { Diabetes mellitus } \\
\text { Hypothyroidism } \\
\text { Trigger fingers } \\
\text { de Quervain's disease } \\
\text { Bilateral medial epicondylitis }\end{array}$ & $\begin{array}{l}5(6.49 \%) \\
1(1.29 \%) \\
6(7.79 \%) \\
2(2.59 \%) \\
1(1.29 \%)\end{array}$ \\
\hline $\begin{array}{l}\text { EDS findings: } \\
\text { Moderate CTS } \\
\text { Severe CTS }\end{array}$ & $\begin{array}{l}34(44.15 \%) \\
43(55.84 \%)\end{array}$ \\
\hline $\begin{array}{l}\text { Postoperative complications observed: } \\
\text { Superficial epidermolysis/ delayed wound healing } \\
\text { Scar tenderness } \\
\text { Pillar pain } \\
\text { Scar hypertrophy }\end{array}$ & $\begin{array}{l}7(9.09 \%) \\
6(7.79 \%) \\
5(6.49 \%) \\
2(2.59 \%)\end{array}$ \\
\hline $\begin{array}{l}\text { Symptomatic relief at } 4 \text { months: } \\
\text { Symptoms improved / relieved } \\
\text { Persistent severe symptoms }\end{array}$ & $\begin{array}{c}74(96.10 \%) \\
3(3.89 \%)\end{array}$ \\
\hline $\begin{array}{l}\text { Preoperative BCTQ Scores: } \\
\text { Mild } \\
\text { Moderate } \\
\text { Severe }\end{array}$ & $\begin{array}{c}0 \\
41(53.24 \%) \\
36(46.75 \%)\end{array}$ \\
\hline $\begin{array}{l}\text { Postoperative BCTQ Scores: } \\
\text { Mild } \\
\text { Moderate } \\
\text { Severe }\end{array}$ & $\begin{array}{c}74(96.10 \%) \\
0\end{array}$ \\
\hline $\begin{array}{l}\text { Age: } 26-69 \text { years. } \\
\text { Duration: } 3-17 \text { months } \\
\text { Recurrence at one year: }\end{array}$ & $\begin{array}{c}3(3.89 \%) \\
\text { Mean } 41.01 \pm 11.70 \text { years. } \\
\text { Mean } 8.94 \pm 5.58 \text { months } \\
0(0 \%)\end{array}$ \\
\hline
\end{tabular}


nerve that increase the volume within the tunnel; for example, space occupying lesions; (3) extrinsic factors that alter the contour of the tunnel; for example, distal radius fracture; (4) neuropathic factors; for example, diabetes mellitus; and (5) the manual workers whose jobs involve daily long hours of forceful repetitive movements of the wrist are at high risk for developing CTS. Use of computers has been reported not to pose any such risk $(2,11,13$ 15).

In this study 6 patients had associated trigger fingers. Serna IC et al reported trigger finger in the same neuropathic hand among 36.1\% individuals. Khanghah YS et al found trigger finger among $26.3 \%$ patients who had undergone carpal tunnel release previously $(11,16)$.

In this study all patients underwent EDS before undergoing surgery. Electrodiagnostic studies not only confirm the diagnosis of CTS particularly among the atypical cases but also help to exclude other conditions, such as polyneuropathy and radiculopathy. They also determine the severity of CTS, which in turn helps to estimate the likely prognosis. Repeat EDS showing improvement also help to reassure the operated patients. The EDS have a sensitivity of $56 \%$ to $85 \%$ and specificity of $94 \%$ to $99 \%$. These can be normal in up to one-third of patients with classic symptoms of CTS. The operating surgeon should carefully read the EDS report to get an exact idea of the motor and sensory loss in median nerve and correlate these findings clinically with the duration of symptoms (especially if long standing) and any thenar muscle atrophy or presence of trophic ulcers. This helps to prognosticate the condition and prudently counsel the patient that the operation will stop further progression and worsening of the condition; however, the sensory motor recovery will depend on nerve regeneration over a period of months $(2,5,17,18)$.

In this study modified Kaplan's lines were employed to serve as a guide for making the incision and safeguarding the nearby coursing vital neurovascular structures (Fig. 1). Kaplan EB described the famous Kaplan's cardinal line to guide hand surgeons regarding key anatomic structures in the palm. The original description given in 1953 has undergone several modifications to encompass all important topographical landmarks of the palm (6).

In this study open carpal tunnel release was performed as a day care procedure. The key step of the procedure is to ensure complete division of the TCL under direct visualization. This in turn results in mechanical decompression of the median nerve and the carpal canal. Equally critical is to prevent iatrogenic injuries to the neurovascular structures and tendons that course through the tunnel. The published literature shows considerably varied practice regarding the surgical approach for releasing TCL. In order to have less incisional discomfort and better scar and expeditious recovery, a variety of incision modifications have been introduced from time to time; for instance, a transverse incision at the wrist crease, mid palmar incision, incision along or parallel to the thenar crease, and mini-incisions. Shorter incisions generally yield relatively better patients' satisfaction and are associated with earlier return to work $(2,3,10,19-25)$.

In this study the mini skin incision was made $6 \mathrm{~mm}$ to
$10 \mathrm{~mm}$ ulnar to the thenar crease. This incision ensured safeguarding the palmar cutaneous branch of median nerve as well as the recurrent motor branch of the median nerve. Also, the subsequent healing tissue and scarring remained well away from the underlying median nerve, thus avoiding one possible cause of persistent or recurrent CTS. The incision did not cross the transverse wrist crease. This approach of skin incision location is akin to Mackinnon SE who employs an incision $6 \mathrm{~mm}$ ulnar to the thenar crease (2).

In this study, the TCL was divided on its most ulnar side of the median nerve. Eroğlu A et al also similarly advocated division of the TCL on the ulnar side of median nerve to avoid the possibility of iatrogenic injuries to the motor and sensory branches arising from the median nerve (21).

In this study except for 3 patients, all had relief of symptoms at 4 months after surgery. In the published literature the overall success rate of open carpal tunnel release is reported to be $\geq 95 \%$. All the patients in this study who continued to have persistent symptoms had diabetes mellitus of more than 5 years duration. Factors responsible for failure of good outcome of TCL release continue to be explored; however, diabetes mellitus, obesity and cervical spine disorders are known to exert negative impact on the outcome of surgery among CTS patients $(26,27)$.

In this study no critical intraoperative complications were encountered. A variety of possible intraoperative complications have been reported in the literature; for instance, incomplete release of TCL, nerve injuries to the palmar cutaneous branch of median nerve, recurrent motor branch of median nerve, median nerve itself, inadvertent entry to Guyon's canal and ulnar nerve injury, and common digital nerves, injury to the superficial palmar arch of ulnar artery and flexor tendon injury. For open carpal tunnel release, the reported incidence of the aforementioned complications involving critical injuries to nerves, arteries or tendons is $0.49 \%(3,28)$.

In this study, 5 patients continued to have pillar pain through their rehabilitation phase. The condition is characterized by variably described pain sensation in the region between the thenar and hypothenar eminences of the palm. The exact etiology is not known. Numerous explanations have been presented in the published literature to explain the causation; for instance, structural alterations in carpal tunnel following the release, muscle and tendon effects, neurogenic factors, and edematous effects. The incidence is reported to be $6 \%$ to $36 \%(3,29-31)$.

In this study no case of recurrence was observed at 1 year follow-up. The published literature has described 3 peculiar categories of conditions that may be encountered in the aftermath of TCL release. These include persistent, recurrent and a newly evolved CTS. Persistent CTS describes the persistence of preoperative symptoms with little or no subjective improvement. It may be due to incomplete release of the TCL, incorrect primary diagnosis, or associated comorbids. Recurrent CTS means that the primary release was initially successful in relieving symptoms, but the primary symptoms have recurred after a period of months to years. The cause of this may be uncon- 
trolled diabetes mellitus or scarring. In case of newly evolved CTS, the patient may complain of even exaggerated symptoms of pain or different symptoms immediately following the surgery. The cause is usually an iatrogenic nerve injury. Incomplete release of the TCL and scar adherence of the median nerve to overlying structures is commonly found among all cases of the persistent, recurrent and newly evolved CTS (32-34).

In this study no additional procedures were instituted as an adjunct to the standard open carpal tunnel release. All the cases were of primary release. Some authors have employed adjunct procedures particularly in cases of secondary TCL release; for instance, tenosynovectomy, internal neurolysis, external neurolysis, epineurotomy, segmental neurectomy, and nerve repair (35).

\section{Cnoclusion}

CTS predominantly affected women aged 30 to 45 years. The open carpal tunnel release with mini-incision yielded good results in terms of relieving symptoms among the majority of patients and was associated with no critical complications or recurrence at one year.

\section{Conflict of Interests}

The authors declare that they have no competing interests.

\section{References}

1. Pourmemari MH, Heliovaara M, Juntura VE, Shiri R. Carpal tunnel release: Lifetime prevalence, annual incidence and risk factors. Muscle Nerve. 2018;58:497-502.

2. Mackinnon SE, Novak CB. Compression neuropathies. In: Wolfe SW, Hotchkiss RN, Pederson WC, Kozin SH, Cohen MS, editor(s). Green's operative hand surgery. 7th edition. Elsevier, Inc. Philadelphia, PA 19103-2899, 2017, p. 921-958.

3. Santosa KB, Chung KC, Waljee JF. Complications of compressive neuropathy: Prevention and management strategies. Hand Clin. 2015;31:139-149.

4. Graham B, Regehr G, Naglie G, Wright JG. Development and validation of diagnostic criteria for carpal tunnel syndrome. J Hand Surg Am. 2006;31:919-24.

5. Werner RA, Andary M. Electrodiagnostic evaluation of carpal tunnel syndrome. Muscle Nerve. 2011;44:597-607.

6. Riordan DC, Kaplan EB. Surface anatomy of the hand and wrist. In: Spinner M (editor). Kaplan's functional and surgical anatomy of the hand. 3rd edition. Philadelphia: JB Lippincott Co. 1984, pp 353-360.

7. Levine DW, Simmons BP, Koris MJ, Daltroy LH, Hohl GG, Fossel $\mathrm{AH}$, et al. A self-administered questionnaire for the assessment of severity of symptoms and functional status in carpal tunnel syndrome. J Bone Joint Surg Am. 1993; 75: 1585-92.

8. Hassankhani GG, Moradi A, Birjandinejad A, Vahedi E, Kachooei AR, Ebrahimzadeh MH. Translation and validation of the Persian version the Boston Carpal Tunnel Syndrome Questionnaire. Arch Bone Jt Surg. 2018; 6: 71-77.

9. Xu L, Huang F, Hou C. Treatment for carpal tunnel syndrome by coronal Z-type lengthening of the transverse carpal ligament. J Pak Med Assoc. 2011; 61: 1068-1071.

10. Keser N, Dortcan N, Cikla U, Uluc K, Celikoglu E, Is M, et al. Semivertical incision: an aesthetically and electrophysiologically effective mini-incision technique for carpal tunnel decompression. Med Sci Monit. 2017; 23: 2993-3000.

11. Serna IC, Garcia-Espinoza JA, Cahuana-Quispe AI, MarquezEspriella C, Cuervo-Vergara MA, Martinez-Lopez JC, Dávila-Diaz R. Diagnosis and treatment of carpal tunnel syndrome in a tertiary care center in Mexico city. World J Plast Surg. 2020;9:219-224.

12. Keir PJ, Rempel DM. Pathomechanics of peripheral nerve loading. Evidence in carpal tunnel syndrome. J Hand Ther. 2005;18:259-69.

13. MacDermid JC, Doherty T. Clinical and electrodiagnostic testing of carpal tunnel syndrome: a narrative review. J Orthop Sports Phys Ther. 2004;34:565-88.

14. Adamson CH, Eisen EA, Kapellusch J, Garg A, Hegmann KT, Thiese MS, et al. Biomechanical risk factors for carpal tunnel syndrome: a pooled study of 2474 workers. Occup Environ Med. 2015;72:33-41.

15. Mediouni Z, Bodin J, Dale M, Herquelot E, Carton M, Leclerc A, et al. Carpal tunnel syndrome and computer exposure at work in two large complementary cohorts. BMJ Open. 2015;5:e008156.

16. Khanghah YS, Akbari H, Bagheri N. Prevalence of carpal tunnel release as a risk factor of trigger finger. World $\mathrm{J}$ Plast Surg. 2020;9:174-178.

17. Jablecki CK, Andary MT, Floeter MK, et al. Practice parameter: electrodiagnostic studies in carpal tunnel syndrome. Report of the American Association of Electrodiagnostic Medicine, American Academy of Neurology, and the American Academy of Physical Medicine and Rehabilitation. Neurology. 2002; 58: 1589-1592.

18. Witt JC, Hentz JG, Stevens JC. Carpal tunnel syndrome with normal nerve conduction studies. Muscle Nerve. 2004; 29: 515-522.

19. Taleisnik J. The palmar cutaneous branch of the median nerve and the approach to the carpal tunnel. An anatomical study. J Bone Joint Surg Am. 1973;55:1212-7.

20. Aslani HR, Alizadeh K, Eajazi A, Karimi A, Karimi MH, Zaferani $\mathrm{Z}$, et al. Comparison of carpal tunnel release with three different techniques. Clin Neurol Neurosurg. 2012;114:965-8.

21. Eroğlu A, Sar E, Topuz AK, Şimşek H, Pusat S. Recurrent carpal tunnel syndrome: evaluation and treatment of the possible causes. World J Clin Cases. 2018; 6: 365-372.

22. Scholten RJPM, Mink van der Molen A, Uitdehaag BMJ, Bouter LM, de Vet HCW. Surgical treatment options for carpal tunnel syndrome. Cochrane Database of Systematic Reviews. 2007: CD003905.

23. Levis CM, Tung TH, Mackinnon SE. Variations in incisions and postoperative management in carpal tunnel surgery. Can J Plast Surg. 2002;10:63-67.

24. Korkmaz M, Ekici MA, Çepoglu MC, Öztürk H. Mini transverse versus longitudinal incision in carpal tunnel syndrome. J Coll Physic Surg Pakistan. 2013, 23: 645-648.

25. Chen, Y, Ji W, Li T, Cong X, Chen Z. The mini-incision technique for carpal tunnel release using nasal instruments in Chinese patients. Medicine. 2017;96:31

26. Karthik K, Nanda R, Stothard J. Recurrent carpal tunnel syndrome-analysis of the impact of patient personality in altering functional outcome following a vascularised hypothenar fat pad flap surgery. J Hand Microsurg. 2012; 4:1-6.

27. Becker J, Nora DB, Gomes I, Stringari FF, Seitensus R, Panosso JS, et al. An evaluation of gender, obesity, age and diabetes mellitus as risk factors for carpal tunnel syndrome. Clin Neurophysiol. 2002;113:1429-34

28. Benson LS, Bare AA, Nagle DJ, Harder VS, Williams CS, Visotsky JL. Complications of endoscopic and open carpal tunnel release. Arthroscopy. 2006;22:919-24.

29. Brooks JJ, Schiller JR, Allen SD, Akelman E. Biomechanical and anatomical consequences of carpal tunnel release. Clin Biomech (Bristol, Avon). 2003;18:685-693.

30. Morrell NT, Harris A, Skjong C, Akelman E. Carpal tunnel release: Do we understand the biomechanical consequences? J Wrist Surg. 2014;3:235-238.

31. Seitz WH Jr, Lall A. Open carpal tunnel release with median neurolysis and Z-plasty reconstruction of the transverse carpal ligament. Curr Orthop Pract. 2013;24:53-57.

32. Tung TH, Mackinnon SE. Secondary carpal tunnel surgery. Plast Reconstr Surg. 2001; 107:1830-43.

33. Zieske 1, Ebersole GC, Davidge K, Fox I, Mackinnon SE. Revision carpal tunnel surgery: A 10-year review of intraoperative findings and outcomes. J Hand Surg Am. 2013; 38:1530-9.

34. Jones NF, Ahn HC, Eo S. Revision surgery for persistent and recurrent carpal tunnel syndrome and for failed carpal tunnel release. Plast Reconstr Surg. 2012; 129:683-692.

35. Mersa B, Saçak B, Kabakafl F, Sezer I, Purisa H, Uluda S. A descriptive study of 972 consecutive cases of carpal tunnel release: role of ancillary procedures. Hand Microsurg. 2013;2:18-22. 